\title{
Otimização do sistema de abastecimento de água da Barragem de Jucazinho através de programação linear
}

O crescente aumento das demandas hídricas nas cidades, geradas pelo crescimento populacional, associados a degradação ambiental, perdas d'água nos sistemas de abastecimento, baixos índices pluviométricos e falhas nos sistemas de gestão, têm resultado em crises hídricas no país, requerendo dos órgãos responsáveis, técnicas capazes de sanar os problemas com o déficit hídrico, de modo que o uso e a preservação desse recurso ocorram de maneira regular. Diante desse contexto, o presente trabalho objetivou otimizar a distribuição de água, monitorar o comportamento das vazões a partir da otimização, com a elaboração de cenários e verificar o consumo nas cidades abastecidas pelo Sistema de Abastecimento de Água da Barragem de Jucazinho - SAABJ. A Barragem de Jucazinho está localizada na cidade de Surubim-PE, as margens do Rio Capibaribe, este sistema é responsável pelo abastecimento de 15 munícipios do agreste pernambucano. A otimização do SAABJ foi realizada utilizando Programação Linear (PL), por meio do método SIMPLEX. Verificou-se que o sistema em estudo, funcionando sob as condições de restrição do regime de atendimento impostas, tem sido ineficiente para atender hidricamente os municípios de sua responsabilidade. Para que o sistema funcione de maneira eficiente, regular e otimizada, as localidades da zona rural de 6 municípios não podem ser atendidas pelo mesmo, e referente a zona urbana, deve-se haver uma redução de $98,09 \%$ no valor de vazão destinado a cidade de Caruaru, o que evidencia a situação crítica de atendimento destas demandas apenas por esta fonte hídrica.

Palavras-chave: Crises hídricas; Otimização; Programação Linear; SIMPLEX.

\section{Optimization of the Jucazinho Dam water supply system through linear programming}

\begin{abstract}
The growing increase in water demands in cities, generated by population growth, associated with environmental degradation, water losses in supply systems, low rainfall and failures in management systems, have resulted in water crises in the country, requiring the responsible agencies. , techniques that can solve the problems with the water deficit, so that the use and preservation of this resource occur regularly. Given this context, the present work aimed to optimize the water distribution, monitor the flow behavior from the optimization, with the elaboration of scenarios and verify the consumption in the cities supplied by the Jucazinho Dam Water Supply System - SAABJ. The Jucazinho Dam is located in the city of Surubim-PE, on the banks of the Capibaribe River, this system is responsible for the supply of 15 municipalities of the Pernambuco agreste. The optimization of the SAABJ was performed using Linear Programming (PL) through the SIMPLEX method. It was found that the system under study, operating under the conditions of restriction of the imposed care regime, has been inefficient to hydraulically serve the municipalities under its responsibility. For the system to function efficiently, regularly and optimally, the rural areas of 6 municipalities cannot be serviced by it, and for the urban area, there must be a reduction of $98.09 \%$ in the amount of flow intended the city of Caruaru, which highlights the critical situation of meeting these demands only by this water source.
\end{abstract}

Keywords: Water problems; Optimization; Linear Programming; SIMPLEX.

Topic: Engenharia de Recursos Hídricos

Reviewed anonymously in the process of blind peer

Ana Carine de Melo Silva (io

Centro Universitário do Vale do Ipojuca, Brasil

http://lattes.cnpq.br/4387606795501810

http://orcid.org/0000-0002-1309-4857

carinemelo01@gmail.com

Cícero Fellipe Diniz de Santana (ib)

Universidade Federal de Campina Grande, Brasil

http://lattes.cnpq.br/7916729062952119

http://orcid.org/0000-0002-9228-3903

cicero.esa@hotmail.com

Virgínia da Costa Brito (iD

Universidade Federal de Campina Grande, Brasil

http://lattes.cnpq.br/3293234957426500

http://orcid.org/0000-0002-5776-0612

virginia.dcb@gmail.com
Received: 12/08/2019

Approved: 27/09/2019

\author{
Eduardo Cabral da Silva (id \\ Universidade Federal de Pernambuco, Brasil \\ http://lattes.cnpq.br/2609068900467599 \\ http://orcid.org/0000-0001-6970-387X \\ eduardo.silva@unifavip.edu.br \\ Rúbia Rafaela Ferreira Ribeiro (iD \\ Universidade Federal de Campina Grande, Brasil \\ http://lattes.cnpq.br/6543797252843494 \\ http://orcid.org/0000-0003-0438-7508 \\ rubiarafaela@hotmail.com \\ Gustavo Euclides da Silva Pereira (iD \\ Centro Universitário do Vale do Ipojuca, Brasil \\ http://lattes.cnpq.br/9471883592403049 \\ http://orcid.org/0000-0002-4997-2471 \\ gustavoesp.1@hotmail.com
}

\section{Referencing this:}

SILVA, A. C. M.; SANTANA, C. F. D.; BRITO, V. C.; SILVA, E. C.; RIBEIRO, R. R. F.; PEREIRA, G. E. S.. Otimização do sistema de abastecimento de água da Barragem de Jucazinho através de programação linear. Revista Ibero Americana de Ciências Ambientais, v.10, n.5, p.101113, 2019. DOI: http://doi.org/10.6008/CBPC21796858.2019.005.0010 


\section{INTRODUÇÃO}

Dentre os recursos naturais, a água foi um elemento considerado em tempos remotos e de cenários hídricos com maior disponibilidade, como um elemento infinito, mas que atualmente possui maior importância como um elemento fundamental à vida, geograficamente restrito e um bem econômico valorizado no mercado. A água para abastecimento humano está cada vez mais rara em todo o mundo, até mesmo em regiões abundantes em termos de disponibilidade hídrica (SOUZA, 2013). Como forma de controle da distribuição adequada de água, a fim de atender ao direito humano essencial de acesso a água potável, definido pela Organização das Nações Unidas (ONU), tem-se os Sistemas de Abastecimento de Água (SAA), que objetivam distribuir água nos padrões exigidos para fins de consumo domésticos, industriais e demais atividades desenvolvidas nas localidades (DUARTE, 2016).

Contudo, devido ao crescimento populacional e desenvolvimento das cidades, gerando uma crescente demanda por água, aliados a intensificada degradação ambiental, uso irracional da água e perdas, os SAA têm sido danificados, chegando a estados de ineficiência (ANDRADE SOBRINHO et al., 2016). Pode-se considerar ainda como fatores contribuintes, as reduções nos índices pluviométricos anuais e as características climáticas das regiões brasileiras (SILVA et al., 2013).

Como forma de controle e uso racional dos recursos hídricos dentre as adversidades existentes, a otimização destaca-se por ser uma metodologia que possibilita a obtenção da melhor solução que garante o nível mínimo de água para o consumo humano e animal, além de suprir as necessidades das atividades relacionadas aos aspectos econômicos e ao desenvolvimento das regiões.

Assim, inserido nesse contexto introdutório, referente a crises hídricas e lapsos quanto a distribuição de água, tem-se o Sistema de Abastecimento de Água da Barragem de Jucazinho (SAABJ), este sistema tem enfrentado adversidades quanto ao acúmulo de água em seu reservatório e na distribuição regular de água entre os municípios de sua responsabilidade.

Proveniente da busca por soluções referentes ao sistema citado, este trabalho objetivou otimizar o consumo e a distribuição de água tratada dos municípios atendidos pelo reservatório de Jucazinho, além de monitorar o comportamento das vazões a partir da otimização, com a elaboração de cenários, a fim de minimizar as perdas e maximizar a eficiência do sistema e a alocação do recurso hídrico. Pode-se destacar através deste estudo, que a determinação de uma hierarquização no abastecimento de água de acordo com as necessidades e prioridades das cidades, o balanceamento na distribuição e a otimização do recurso hídrico, bem como dos processos envolvidos no SAA, se usados dentro do planejamento e gestão das bacias hidrográficas podem contribuir para o desenvolvimento satisfatório e regular do sistema da barragem de Jucazinho, afetando de forma positiva as áreas econômicas, ambientais e sociais.

Para a otimização do SAABJ, foi utilizada a Programação Linear (PL), com a aplicação do método SIMPLEX. Com o resultado da otimização observou-se que este sistema sob as condições impostas mostrase ineficiente para atender aos 15 municípios de sua responsabilidade, principalmente às zonas rurais de 6 cidades e à zona urbana de Caruaru/PE. 


\section{REVISÃO TEÓRICA}

De acordo com Pinto (2015), devido ao grande volume de escoamento superficial médio das precipitações naturais ao longo do país, o Brasil não deveria ter desafios relacionados a gestão hídrica. Contudo, esse escoamento não é homogêneo entre as regiões. Dessa forma, em conjunto com a crescente demanda por água para atividades econômicas, para a produção de energia e para o consumo e higiene, associados às mudanças climáticas, a poluição das fontes hídricas, a inexistência da preservação dos mananciais e a ausência de conscientização quanto ao uso adequado e sem desperdícios, têm gerado déficits na distribuição e disponibilidade do recurso hídrico no país.

Na região Nordeste, as características naturais, com altas temperaturas, forte insolação e altas taxas de evapotranspiração, fazem com que a região sofra com a baixa disponibilidade hídrica (BRAZÃO et al., 2016). De acordo com Silva et al. (2017), sabe-se ainda que a região é tradicionalmente afetada por secas prolongadas seguidas de inundações provocadas pelo caráter torrencial e concentrado das chuvas.

Desta forma, para controlar a distribuição hídrica, nos municípios da região, são construídos os SAA, seus materiais e equipamentos, destinados a produção e distribuição de água potável para as populações (PEREIRA, 2017). Contudo, um fator crítico que envolve os SAA no Brasil é o percentual de perdas, pois o país apresenta níveis elevados na distribuição. Os principais motivos são a depreciação das tubulações, bem como a falta de uma política que vise a modernização dos sistemas existentes (DIEESE, 2016).

Inserido no contexto de perdas e conflitos entre demandas e disponibilidades hídricas nos diversos usos da água, os modelos de otimização são ferramentas que promovem o melhor atendimento as demandas e redução das perdas econômicas. Através da otimização, também é possível se obter um melhor uso dos recursos hídricos disponíveis e a redução dos prejuízos advindos da escassez de água (MATTIUZI, 2018).

\section{METODOLOGIA}

\section{Área de estudo}

A área de estudo é a bacia hidrográfica do Rio Capibaribe, possuindo como amostra a barragem de Jucazinho e as 15 cidades abastecidas por esse SAA, tendo em vista que a barragem em estudo está inserida na bacia citada. De acordo com a APAC (Agência Pernambucana de Água e Clima), a bacia hidrográfica do Rio Capibaribe corresponde a unidade de planejamento hídrico UP2 e está localizada na porção norte-oriental

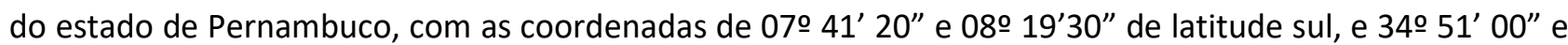
360 41' 58" de longitude oeste.

A bacia possui uma área de abrangência de $7.454,88 \mathrm{~km}^{2}$, o equivalente a 7,58\% do estado e abrange uma totalidade de 42 municípios do estado de Pernambuco, dentre esses alguns estão totalmente inseridos na bacia, outros apenas parcialmente e alguns com sede na mesma. De acordo com o documento emitido pelo Tribunal de Contas da União (TCU), a barragem de Jucazinho fica situada no município de Surubim no estado de Pernambuco, as margens do Rio Capibaribe, o qual é represado pela barragem. Possui uma distância média da capital do estado, Recife, de $120 \mathrm{~km}$. A mesma foi inaugurada no ano 2000. 
De acordo com a Compesa (2017), a barragem possui uma capacidade total de reserva de 327 milhões de $\mathrm{m}^{3}$, é responsável pelo abastecimento de 15 cidades do estado, que são elas: Santa Cruz do Capibaribe, Riacho das Almas, Cumaru, Passira, Salgadinho, Casinhas, Surubim, Vertentes, Vertente do Lério, Santa Maria do Cambucá, Frei Miguelinho, Toritama, Caruaru, Bezerros e Gravatá, além de diversos distritos e povoados dos 15 municípios que fazem parte do sistema integrado. Sua capacidade de produção é de 1.300 I/s, atendendo a uma população de aproximadamente 800 mil pessoas ao longo de seus 206 quilômetros de adutoras.

Pedrosa (2015), afirma ainda que o SAABJ se distribui por duas linhas principais, que são elas, tramo norte e tramo sul. O tramo norte é responsável pelo abastecimento dos municípios de Salgadinho, Casinhas, Surubim, Vertente do Lério, Santa Maria do Cambucá, Frei Miguelinho, Vertentes, Toritama e Santa Cruz do Capibaribe. Enquanto o tramo sul abastece às cidades de Cumaru, Passira, Gravatá, Bezerros, Riacho das Almas e Caruaru (GOMES, 2010). A Figura 1 apresenta uma imagem de satélite acerca da interligação entre os municípios abastecidos pelo SAABJ. Por conseguinte a Figura 2 apresenta a esquematização do SAABJ e a distribuição da água que é efetuada a partir da barragem, expondo os nós e bifurcações.

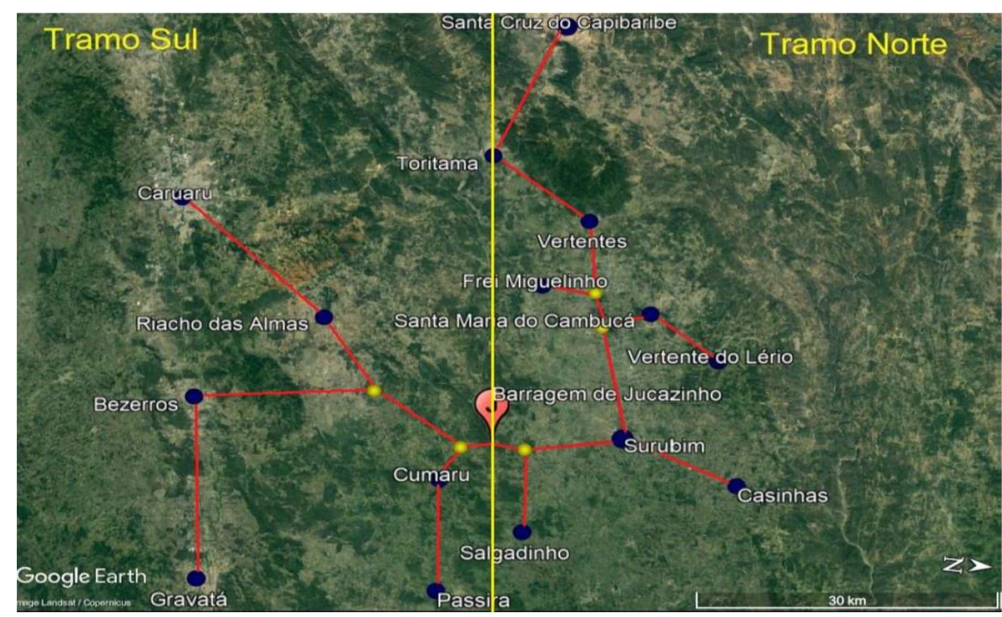

Figura 1: Imagem de satélite da interligação entre cidades do SAABJ.

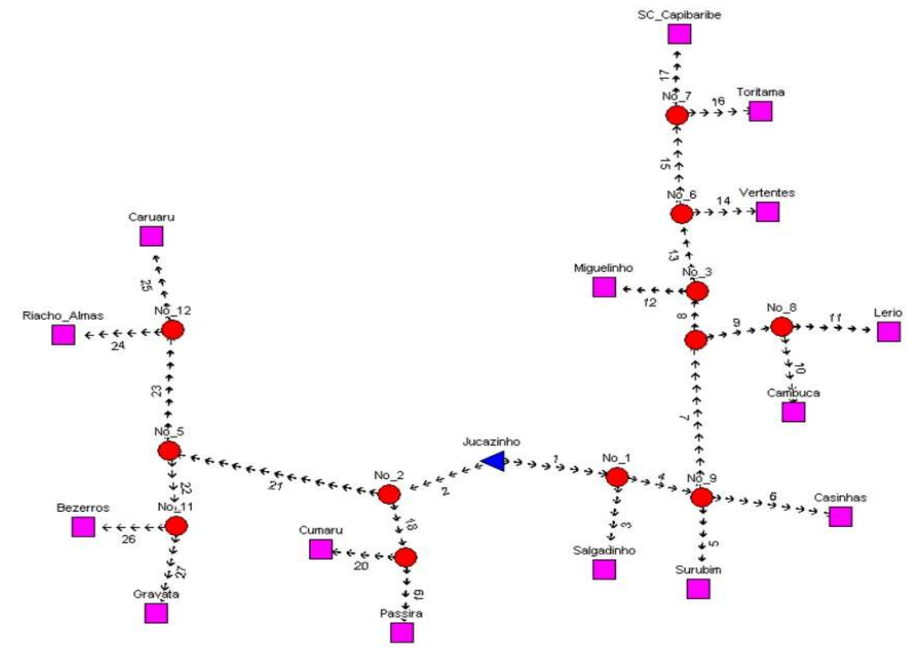

Figura 2: Esquematização do SAABJ.

\section{Otimização: Método SIMPLEX}

O método SIMPLEX é um método iterativo, que determina numericamente a solução ótima de um 
modelo de PL (MANSILHA et al., 2017). A partir de uma solução básica inicial, escolhe outras soluções para teste, tornando o percurso até a solução ótima, mais curto (SILVA, 2016). De acordo com Mansilha et al. (2017), para possível solução do problema, ele deve estar escrito na forma padrão e sujeito a algumas restrições, de acordo com o formato matricial. O formato padrão de um problema de programação linear, escrito em formato matricial, resulta em uma apresentação mais compacta. A Figura 3, mostra a sequência de passos seguida para utilização do método SIMPLEX, no presente estudo.

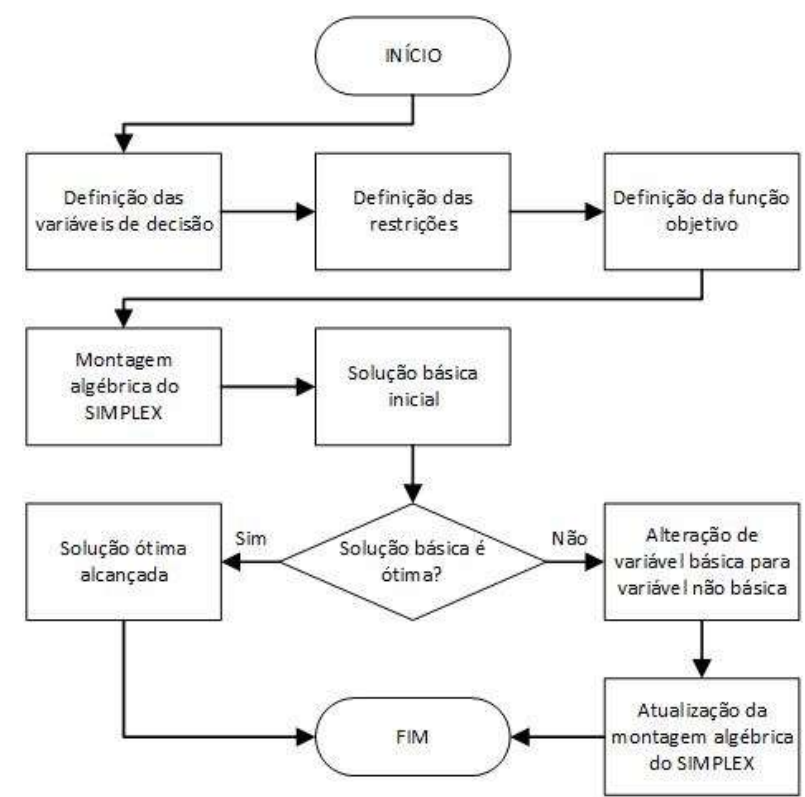

Figura 3: Fluxograma do método SIMPLEX.

Para aplicação do SIMPLEX no presente estudo, foram obtidas as variáveis necessárias ao mesmo. Foi realizado um levantamento das cidades abastecidas pelo reservatório de Jucazinho e verificado o consumo total de água por ano das cidades, através do Sistema Nacional de Informações sobre Saneamento (SNIS). Tendo em vista que o SNIS possui uma defasagem de 2 anos, foi possível obter o consumo de água referente ao ano de 2016.

Posteriormente, a partir do último censo demográfico realizado pelo IBGE, no ano de 2010, obtevese o quantitativo populacional total, urbano e rural das cidades. A partir desses dados foi calculada a equivalência da população urbana em relação a total, e a equivalência da população rural em relação a total e posteriormente o consumo de água parcial das cidades $\mathrm{em}^{3} / \mathrm{s}$, o consumo de água referente a ambas populações.

Com a obtenção dos volumes parciais de água consumidos de cada cidade, foi atribuída uma prioridade ao atendimento de água no valor de 100\% para área urbana e um valor de prioridade de 50\% para área rural. Ao considerar que a zona rural pode ser abastecida através de fontes alternativas, esta atribuição foi de fundamental importância para a aplicação do método.

Para complementar os dados necessários a aplicação, foram adquiridos dados referentes as vazões destinadas às cidades a partir do reservatório, obtidos junto à Companhia Pernambucana de Saneamento (Compesa). Conforme os dados, cidades de maior porte e demanda, como Surubim e Caruaru, recebiam um valor aproximado de vazão entre 60 a 80 L/s, enquanto as demais cidades do Tramo Norte e do Tramo Sul, 
não excediam 10 L/s. Por convenção, as demandas atribuídas para Caruaru e Surubim, foram as vazões de $80 \mathrm{~L} / \mathrm{s}$ respectivamente, enquanto as demais cidades, receberam $9 \mathrm{~L} / \mathrm{s}$.

\section{Formulação da função objetivo e das restrições}

Para a aplicação do método SIMPLEX foram necessárias as formulações das variáveis de decisão, das restrições e da função objetivo. Com a função objetivo, obtida a partir da análise do SAABJ, foi verificado o percurso do abastecimento até as demandas para análise da ordem em que os municípios são atendidos. A função objetivo foi de maximização e está sendo representada pela Equação 1.

Deve-se ressaltar que, a justificativa para os valores numéricos (10 e 5) utilizados, é devido a hierarquização criada na distribuição de água entre os municípios, sendo o valor 10 atribuído a população urbana, e o valor 5 a população rural. Todas equações e resoluções referentes ao método foram formuladas e solucionadas em ambiente Microsoft Excel, utilizando a ferramenta Solver. Deve-se destacar que as siglas $\mathrm{C} 1, \mathrm{C} 2, \mathrm{C} 3, \ldots, \mathrm{Cn}$, referem-se às cidades, $\mathrm{N} 1, \mathrm{~N} 2, \mathrm{~N} 3, \ldots, \mathrm{Nn}$, aos nós, enquanto que as letras $\mathrm{U}$ e $\mathrm{R}$, à urbana e rural, respectivamente.

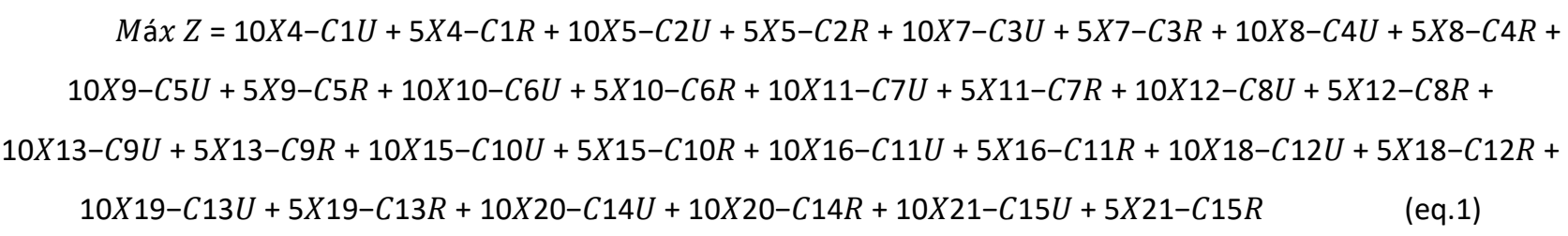

\section{Elaboração de cenários para monitoramento das vazões}

Para melhores análises do sistema de distribuição de água, foram elaborados 4 cenários para monitorar o atendimento das vazões requeridas pelos municípios, com acréscimos e decréscimos nas demandas e redução na vazão de saída do reservatório de Jucazinho. Foram aplicadas reduções de $25 \%$ e $50 \%$ na vazão a jusante do reservatório, de $25 \%$ no consumo de água urbano e de $25 \%$ no consumo de água apenas das cidades que compõem o Tramo Norte.

A partir dos cenários, que diferem das condições reais sob o qual o sistema havia sido imposto para a realização da otimização, foi possível monitorar o comportamento das vazões e verificar se existiam divergências quanto as condições de restrições iniciais do sistema. Torna-se importante mencionar que as variáveis de decisão, a função objetivo e as restrições usadas nos cenários, foram as mesmas utilizadas na otimização, com o mesmo esquema de rede e prioridade de abastecimento.

\section{RESULTADOS E DISCUSSÃO}

Como resposta do método SIMPLEX, foram emitidos os relatórios de resposta e sensibilidade para análise e discussão dos dados. Sabe-se que o relatório de resposta divide-se em três partes, mediante a análise da primeira, se obtém o tipo de problema de otimização tratado, de maximização ou minimização, que para o presente estudo foi de maximização, bem como se extrai o valor inicial e final da função objetivo, antes e depois da otimização, enquanto, a segunda parte refere-se às variáveis de decisão. Desta forma, os 
valores da função objetivo e os valores das variáveis de decisão estão expressos nas Tabela 1 e Tabela 2, respectivamente.

Tabela 1: Valores da função objetivo.

\begin{tabular}{|l|l|}
\hline Função Objetivo & Valor Final \\
\hline Valor Original & 2,371129063 \\
\hline $\mathbf{2 , 3 7 1 1 2 9 0 6 3}$ & \\
\hline
\end{tabular}

Tabela 2: Valores das variáveis de decisão.

\begin{tabular}{|c|c|c|c|c|c|c|c|c|}
\hline Municípios & Código & Vazões & Municípios & Código & Vazões & Municípios & Código & Vazões \\
\hline \multirow[t]{2}{*}{ Cumaru } & C1U & $2,50 \mathrm{E}-03$ & Caruaru & C6U & $2,84 \mathrm{E}-01$ & Vertente do Lério & C11U & $7,40 \mathrm{E}-05$ \\
\hline & C1R & $2,85 \mathrm{E}-03$ & & C6R & $3,58 \mathrm{E}-02$ & & C11R & $2,47 \mathrm{E}-04$ \\
\hline \multirow[t]{2}{*}{ Passira } & $\mathrm{C} 2 \mathrm{U}$ & $3,44 \mathrm{E}-03$ & Salgadinho & $\mathrm{C} 7 \mathrm{U}$ & $2,96 \mathrm{E}-04$ & Frei Miguelinho & C12U & $4,05 E-04$ \\
\hline & $\mathrm{C} 2 \mathrm{R}$ & $3,62 \mathrm{E}-03$ & & C7R & $6,03 E-04$ & & C12R & $1,30 \mathrm{E}-03$ \\
\hline \multirow[t]{2}{*}{ Bezerros } & $\mathrm{C} 3 \mathrm{U}$ & $4,29 E-02$ & Surubim & $\mathrm{C} 8 \mathrm{U}$ & $2,75 \mathrm{E}-02$ & Vertentes & C13U & $2,90 \mathrm{E}-03$ \\
\hline & C3R & $7,70 \mathrm{E}-03$ & & $\mathrm{C} 8 \mathrm{R}$ & $9,03 E-03$ & & C13R & $1,18 \mathrm{E}-03$ \\
\hline \multirow[t]{2}{*}{ Gravatá } & $\mathrm{C} 4 \mathrm{U}$ & $1,03 \mathrm{E}-01$ & Casinhas & $\mathrm{C9U}$ & $9,82 \mathrm{E}-05$ & Toritama & C14U & $1,06 \mathrm{E}-02$ \\
\hline & C4R & $1,21 \mathrm{E}-02$ & & C9R & $6,95 \mathrm{E}-04$ & & C14R & $4,45 \mathrm{E}-04$ \\
\hline \multirow[t]{2}{*}{ Riacho } & $\mathrm{C} 5 \mathrm{U}$ & $3,05 E-03$ & Santa Maria do Cambucá & C10U & $5,12 \mathrm{E}-04$ & Santa Cruz do Capibaribe & C15U & $2,69 \mathrm{E}-02$ \\
\hline & $\mathrm{C} 5 \mathrm{R}$ & $3,62 \mathrm{E}-03$ & & C10R & $1,52 \mathrm{E}-03$ & & C15R & $6,25 \mathrm{E}-04$ \\
\hline
\end{tabular}

Por fim, a terceira parte do relatório de resposta refere-se às restrições, a partir desta foi possível identificar em quais cidades as condições de restrições não foram atendidas, essa identificação é realizada através da coluna Status, os pontos que indicam status de Não-associação, representam os pontos não atendidos, consequentemente, a indicação do status de Associação sinaliza o atendimento às restrições. Com a interpretação deste relatório, pôde-se observar que as condições não foram alcançadas em 7 pontos, devese destacar que estes pontos são discutidos numericamente apenas no relatório de sensibilidade. A terceira parte referente ao relatório de resposta pode ser analisada a partir da Tabela 3.

Referente ao relatório de sensibilidade, sua divisão ocorre em duas partes, a primeira está relacionada às mudanças que podem acontecer nos coeficientes das variáveis de decisão da função objetivo, enquanto a segunda, está para as eventuais alterações que as constantes de restrições podem sofrer. 0 relatório de sensibilidade está sendo expresso através da Tabela 4.

Tabela 3: Relatório de Resposta da Otimização.

\begin{tabular}{|l|l|l|l|l|}
\hline Nome & Localidade & Valor da Célula & Fórmula & Status \\
\hline \multirow{4}{*}{ Cumaru } & Pop. Urb. & 0,002504199 & $<=$ & Associação \\
\cline { 2 - 5 } & Pop. Rur. & 0 & $<=$ & Não Associação \\
\hline \multirow{2}{*}{ Bezsira } & Pop. Urb. & 0,003436928 & $<=$ & Associação \\
\cline { 2 - 5 } & Pop. Rur. & 0 & $<=$ & Não Associação \\
\hline \multirow{2}{*}{ Gravatá } & Pop. Urb. & 0,042891667 & $<=$ & Associação \\
\cline { 2 - 5 } & Pop. Rur. & 0 & $<=$ & Não-associação \\
\hline \multirow{2}{*}{ Riachos das Almas } & Pop. Urb. & 0,102694939 & $<=$ & Associação \\
\cline { 2 - 5 } & Pop. Rur. & 0 & $<=$ & Não-associação \\
\hline \multirow{2}{*}{ Caruaru } & Pop. Urb. & 0,003047234 & $<=$ & Associação \\
\cline { 2 - 5 } & Pop. Rur. & 0 & $<=$ & Não-associação \\
\hline \multirow{2}{*}{ Salgadinho } & Pop. Urb. & 0,005425033 & Não-associação \\
\cline { 2 - 5 } & Pop. Rur. & 0 & $<=$ & Não-associação \\
\hline \multirow{2}{*}{ Surubim } & Pop. Urb. & 0,000295603 & $<=$ & Associação \\
\cline { 2 - 5 } & Pop. Rur. & 0,00060337 & $<=$ & Associação \\
\hline \multirow{2}{*}{ Casinhas } & Pop. Urb. & 0,027452564 & $<=$ & Associação \\
\cline { 2 - 5 } & Pop. Rur. & 0,00902638 & $<=$ & Associação \\
\hline \multirow{2}{*}{ Santa Maria do Cambucá } & Pop. Urb. & 0,000098207 & $<=$ & Associação \\
\cline { 2 - 4 } & Pop. Rur. & 0,000695172 & $<=$ & Associação \\
\hline
\end{tabular}




\begin{tabular}{|l|l|l|l|l|}
\hline \multirow{2}{*}{ Vertente do Lério } & Pop. Urb. & 0,0000739708 & $<=$ & Associação \\
\cline { 2 - 4 } & Pop. Rur. & 0,000247249 & $<=$ & Associação \\
\hline \multirow{2}{*}{ Frei Miguelinho } & Pop. Urb. & 0,000405106 & $<=$ & Associação \\
\cline { 2 - 4 } & Pop. Rur. & 0,001303417 & $<=$ & Associação \\
\hline \multirow{2}{*}{ Vertentes } & Pop. Urb. & 0,002899654 & $<=$ & Associação \\
\cline { 2 - 4 } & Pop. Rur. & 0,001183299 & $<=$ & Associação \\
\hline Toritama & Pop. Urb. & 0,010628007 & $<=$ & Associação \\
\cline { 2 - 4 } & Pop. Rur. & 0,00033378975 & $<=$ & Associação \\
\hline Santa Cruz do Capibaribe & Pop. Urb. & 0,0201917025 & $<=$ & Associação \\
\cline { 2 - 4 } & Pop. Rur. & 0,00046897125 & $<=$ & Associação \\
\hline Reservatório de Jucazinho & - & 0,16 & $<$ \\
\hline
\end{tabular}

De acordo com este relatório, a solução ótima para o sistema é expressa na coluna Final Valor, esses valores garantem que o sistema funcione de maneira otimizada e eficiente, representam a solução da otimização. A coluna 'Restrição Lateral R.H' são os consumos de água dos municípios, é o parâmetro de comparação com a solução ótima. As colunas 'Permitido Aumentar' e 'Permitido Reduzir' representam os limites impostos, são os valores que podem ser somados ou subtraídos dos valores inciais de demandas sem que a solução ótima se altere. Equanto que os dados da coluna 'Preço Sombra', representam os acréscimos e decréscimos unitários de vazão ao valor ótimo, dentro dos limites permitidos. Através da análise do relatório de sensibilidade, nota-se que o SAABJ, trabalhando sob as condições reais e impostas pelo estudo, necessita restringir a distribuição de água nas localidades em que os valores de vazão requeridos não foram atendidos para que Jucazinho funcione de maneira otimizada.

Os pontos em que as condições não foram satisfeitas, foram as áreas rurais de Cumaru, Passira, Bezerros, Gravatá, Riacho das Almas e Caruaru, e a área urbana de Caruaru, sendo esta, a única área urbana que não foi atendida. As sedes citadas, nos quais os valores de vazão não foram alcançados, compõem o Tramo Sul, o que significa que nenhum município pertencente ao Tramo Sul está sendo suficientemente atendido em termos hídricos a partir do reservatório de Jucazinho. Enquanto que no Tramo Norte, a situação é divergente, uma vez que todas as circunstâncias sucederam satisfatoriamente, o que indica que o reservatório satisfaz as demandas da população urbana e rural das cidades de Salgadinho, Casinhas, Surubim, Vertente do Lério, Santa Maria do Cambucá, Frei Miguelinho, Vertentes, Toritama e Santa Cruz do Capibaribe.

Tabela 4: Relatório de Sensibilidade da Otimização.

\begin{tabular}{|c|c|c|c|c|c|}
\hline Nome & Final Valor & Sombra Preço & Restrição Lateral R.H. & Permitido Aumentar & Permitido Reduzir \\
\hline \multirow[t]{2}{*}{ Cumaru } & Pop. Urb. & 0,002504 & 0 & 0,002504 & 0,005425 \\
\hline & Pop. Rur. & 0 & 0 & 0,002848 & $1 \mathrm{E}+30$ \\
\hline \multirow[t]{2}{*}{ Passira } & Pop. Urb. & 0,003437 & 0 & 0,003437 & 0,005425 \\
\hline & Pop. Rur. & 0 & 0 & 0,003619 & $1 \mathrm{E}+30$ \\
\hline \multirow[t]{2}{*}{ Bezerros } & Pop. Urb. & 0,042892 & 0 & 0,042892 & 0,005425 \\
\hline & Pop. Rur. & 0 & 0 & 0,007699 & $1 \mathrm{E}+30$ \\
\hline \multirow[t]{2}{*}{ Gravatá } & Pop. Urb. & 0,102695 & 0 & 0,102695 & 0,005425 \\
\hline & Pop. Rur. & 0 & 0 & 0,012123 & $1 E+30$ \\
\hline \multirow[t]{2}{*}{ Riachos das Almas } & Pop. Urb. & 0,003047 & 0 & 0,003047 & 0,005425 \\
\hline & Pop. Rur. & 0 & 0 & 0,003617 & $1 \mathrm{E}+30$ \\
\hline \multirow[t]{2}{*}{ Caruaru } & Pop. Urb. & 0,005425 & 0 & 0,283685 & $1 \mathrm{E}+30$ \\
\hline & Pop. Rur. & 0 & 0 & 0,03584 & $1 \mathrm{E}+30$ \\
\hline \multirow[t]{2}{*}{ Salgadinho } & Pop. Urb. & 0,000296 & 10 & 0,000296 & $1 \mathrm{E}+30$ \\
\hline & Pop. Rur. & 0,000603 & 5 & 0,000603 & $1 \mathrm{E}+30$ \\
\hline \multirow[t]{2}{*}{ Surubim } & Pop. Urb. & 0,027453 & 10 & 0,027453 & $1 \mathrm{E}+30$ \\
\hline & Pop. Rur. & 0,009026 & 5 & 0,009026 & $1 \mathrm{E}+30$ \\
\hline Casinhas & Pop. Urb. & $9,82 \mathrm{E}-05$ & 10 & $9,82 \mathrm{E}-05$ & $1 \mathrm{E}+30$ \\
\hline
\end{tabular}




\begin{tabular}{|l|l|l|l|l|l|}
\hline & Pop. Rur. & 0,000695 & 5 & 0,000695 & $1 \mathrm{E}+30$ \\
\hline Santa Maria do Cambucá & Pop. Urb. & 0,000512 & 10 & 0,000512 & $1 \mathrm{E}+30$ \\
\hline & Pop. Rur. & 0,001523 & 5 & 0,001523 & $1 \mathrm{E}+30$ \\
\hline Vertente do Lério & Pop. Urb. & $7,4 \mathrm{E}-05$ & 10 & $7,4 \mathrm{E}-05$ & $1 \mathrm{E}+30$ \\
\hline & Pop. Rur. & 0,000247 & 5 & 0,000247 & $1 \mathrm{E}+30$ \\
\hline Frei Miguelinho & Pop. Urb. & 0,000405 & 10 & 0,000405 & $1 \mathrm{E}+30$ \\
\hline & Pop. Rur. & 0,001303 & 5 & 0,001303 & $1 \mathrm{E}+30$ \\
\hline Vertentes & Pop. Urb. & 0,0029 & 10 & 0,0029 & $1 \mathrm{E}+30$ \\
\hline & Pop. Rur. & 0,001183 & 5 & 0,001183 & $1 \mathrm{E}+30$ \\
\hline Toritama & Pop. Urb. & 0,010628 & 10 & 0,010628 & $1 \mathrm{E}+30$ \\
\hline & Pop. Rur. & 0,000445 & 5 & 0,000445 & $1 \mathrm{E}+30$ \\
\hline Santa Cruz do Capibaribe & Pop. Urb. & 0,026922 & 10 & 0,026922 & $1 \mathrm{E}+30$ \\
\hline & Pop. Rur. & 0,000625 & 5 & 0,000625 & $1 \mathrm{E}+30$ \\
\hline Reservatório de Jucazinho & - & 0,16 & 10 & 0,16 & 0,27826 \\
\hline
\end{tabular}

Deve-se destacar que o número de habitantes do Tramo Sul é superior a do Tramo Norte, embora o este contabilize um total de 9 cidades, e aquele apenas 6. Todavia, o quantitativo populacional do Tramo Sul é o equivalente a aproximadamente o dobro do Tramo Norte, este contabilizando apenas 258.138 habitantes e aquele 515.011 habitantes. Uma possível justificativa para os resultados encontrados, é que, para que o sistema consiga realizar o atendimento de maneira eficiente, é necessário que o SAABJ oferte água apenas para as zonas urbanas nos municípios de Cumaru, Passira, Bezerros, Gravatá, Riacho das Almas e Caruaru, devido à falta de vazão suficiente para essas demandas, situação representada pelos resultados das variáveis de atendimento das restrições iguais a zero. Já que tratam-se de regiões onde o custo de distribuição é oneroso, uma alternativa viável para as demandas rurais é o suprimento realizado através de outras fontes, além do Jucazinho. Nas condições impostas o atendimento aos valores de vazão impostos para a otimização, não fizeram parte da solução ótima obtida no $S A A B J$, significando que a oferta de água da Barragem, exclusivamente, não é suficiente para atender as demandas rurais das cidades do Tramo Sul.

Em relação ao atendimento à população urbana da cidade de Caruaru, o valor de vazão que pode ser distribuído de acordo com a solução ótima, é de $0,005425 \mathrm{~m}^{3} / \mathrm{s}$, enquanto que o valor requerido é de $0,283685 \mathrm{~m}^{3} / \mathrm{s}$, ou seja, para que o sistema trabalhe corretamente, se faz necessário uma redução de 0,27826 $\mathrm{m}^{3} / \mathrm{s}$ no valor de vazão distribuído para esse município, o equivalente a uma redução de $98,09 \%$. O valor que pode ser distribuído pelo reservatório de Jucazinho é significativamente inferior ao solicitado, representa apenas 1,91\% do valor total de vazão requerido pela zona urbana.

\section{Monitoramento das vazões - Cenários elaborados}

Como reposta do método SIMPLEX para os cenários elaborados, também foram obtidos os relatórios de reposta e sensibilidade referentes a cada cenário para identificação das variações dos resultados e discussão da otimização.

\section{Cenário 1}

Para a elaboração do primeiro cenário, foi convencionado uma redução de $25 \%$ da saída de água do reservatório, mantendo um funcionamento de apenas $75 \%$ de sua capacidade. Foi adotado um valor de 120 L/s, o equivalente a $75 \%$ de 160 L/s, que era a vazão de saída real do sistema. Para esse cenário houve 
alteração apenas na vazão do reservatório, os valores de consumo das cidades se mantiveram inalterados. A Tabela 5 expressa os valores da função objetivo obtidos para o presente cenário, com uma variação de 0,4 entre o valor original e o valor final.

Tabela 5: Valores da função objetivo do Cenário 1.

\begin{tabular}{|l|l|}
\hline Função Objetivo & Valor Final \\
\hline Valor Original & 1,971129063 \\
\hline $\mathbf{2 , 3 7 1 1 2 9 0 6 3}$ & \\
\hline
\end{tabular}

A partir da análise dos relatórios de resposta e sensibilidade emitidos para este cenário, foi observado que o sistema operando com $75 \%$ de sua capacidade de vazão, os pontos em que as condições não foram satisfeitas se mantiveram nas áreas rurais de Cumaru, Passira, Bezerros, Gravatá, Riacho das Almas e Caruaru. Porém, o desatendimento das condições em relação a população urbana, ocorreu nas cidades de Gravatá, Riacho das Almas e Caruaru, diferentemente de quando operou com 100\% de sua capacidade, que não atendeu apenas à Caruaru.

Em relação a zona rural, o sistema não se mostrou eficaz na distribuição de água destinada a essas localidades, pois os resultados ótimos para esses pontos foram iguais a zero, o que significa que o sistema estará funcionando corretamente, se nenhum valor de vazão for enviado para área rural das cidades do Tramo Sul.

Quanto a área urbana, foi evidenciada uma redução de vazão de 31,53 L/s na cidade de Gravatá, o sistema só é capaz de fornecer $71,17 \mathrm{~L} / \mathrm{s}$, o equivalente a $69,3 \%$ da sua demanda total. Com o sistema operando apenas com 75\%, o mesmo se torna irregular em Riacho das Almas e Caruaru, pois o valor ótimo obtido foi igual a zero, ou seja, o sistema não é capaz de atendê-las. Portanto, fica evidenciado que distribuindo apenas um valor de vazão de 120 L/s, o reservatório de Jucazinho não seria suficiente para atender a três cidades completas, população urbana e rural, de Gravatá, Riacho das Almas e Caruaru, o mesmo, ainda se mostra ineficiente para com a população rural dos municípios de Cumaru, Passira e Bezerros.

\section{Cenário 2}

Para o Cenário 2, foi realizada uma redução de $50 \%$ da água destinada para o atendimento da rede, foi imposto que o reservatório estava funcionando apenas com uma vazão de saída de $80 \mathrm{~L} / \mathrm{s}$. Os valores da função objetivo, apresentando uma variação de 0,8, estão expressos na Tabela 6 .

Tabela 6: Valores da função objetivo do Cenário 2.

\begin{tabular}{|l|l|}
\hline Função Objetivo & Valor Final \\
\hline Valor Original & 1,571129063 \\
\hline $\mathbf{2 , 3 7 1 1 2 9 0 6 3}$ & \\
\hline
\end{tabular}

Através dos relatórios, novamente foi verificado um déficit no abastecimento das áreas urbanas e rurais de Gravatá, Riacho das Almas e Caruaru. Houve uma redução de 30,35\% da vazão enviada para a população urbana de Gravatá, o valor obtido pela solução ótima representou apenas $69,65 \%$ do que é requerido pelo município, e nenhum valor de vazão para a área rural, enquanto que para Riacho das Almas 
e Caruaru, o sistema não foi capaz de oferecer nenhum valor de vazão para ambas as áreas. O sistema permaneceu ineficiente para atender as demandas hídricas das localidades rurais de Cumaru, Passira e Bezerros, possuindo um valor igual a zero como solução ótima. Fica constado que novamente as condições não foram satisfeitas apenas no Tramo Sul.

\section{Cenário 3}

Na elaboração do Cenário 3, foram reduzidas as vazões das demandas referentes ao consumo urbano em $25 \%$, em todas as 15 cidades, com o objetivo de avaliar o atendimento à zona rural, a partir do reservatório. Mediante a Tabela 7, é possível verificar os valores da função objetivo, que não se alteraram.

Tabela 7: Valores da função objetivo do Cenário 3.

\begin{tabular}{|l|l|}
\hline Função Objetivo & Valor Final \\
\hline Valor Original & 2,197911531 \\
\hline $\mathbf{2 , 1 9 7 9 1 1 5 3 1}$ &
\end{tabular}

A partir desse cenário, observou-se que o problema ainda persiste perante o atendimento à população rural das cidades que formam o Tramo Sul, bem como da zona urbana de Caruaru, o resultado ótimo para essa área foi apenas $20,71 \%$ do total, representando assim uma redução de $79,29 \%$ do envio de vazão. Enquanto que os resultados alcançados novamente para as áreas rurais foram iguais a zero.

O resultado desse cenário se assemelha ao resultado da otimização do sistema sob condições reais de funcionamento. Pode ser observado que se reduzido $25 \%$ do consumo urbano de água, as zonas rurais das cidades do Tramo Sul ainda continuam sem atendimento, porém em relação a localidade urbana de Caruaru, o sistema se mostra mais eficiente ao indicar um aumento de $18,8 \%$ na distribuição de vazão, pois o mesmo trabalhando com a condição de $100 \%$ de consumo de água era capaz de distribuir apenas o equivalente a 1,91\% do valor total de demanda, enquanto que o sistema funcionando sob a condição de $75 \%$, é capaz de distribuir o equivalente a $20,71 \%$ do valor requerido.

\section{Cenário 4}

No Cenário 4, foi aplicada uma redução de $25 \%$ no consumo de água das cidades do Tramo Norte, que são Salgadinho, Casinhas, Surubim, Vertente do Lério, Santa Maria do Cambucá, Frei Miguelinho, Vertentes, Toritama e Santa Cruz do Capibaribe. É importante destacar que a redução foi aplicada para ambas as populações, rurais e urbanas. Na Tabelas 8, estão expressos os valores da função objetivo, foi possível verificar uma alteração em seu valor, uma redução de 0,019564734.

Tabela 8: Valores da função objetivo do Cenário 4.

\begin{tabular}{|l|l|}
\hline Função Objetivo & Valor Final \\
\hline Valor Original & 2,178346797 \\
\hline $\mathbf{2 , 1 9 7 9 1 1 5 3 1}$ & \\
\hline
\end{tabular}

Diante da solução ótima exposta no Cenário 4, fica constatada mais uma vez a ineficiência do reservatório de Jucazinho para com as zonas rurais do Tramo Sul, pois o resultado obtido nesses pontos foram iguais a zero. Enquanto que, a redução para área urbana de Caruaru, dessa vez foi igual a 98,09\%, 
sendo capaz de distribuir apenas o equivalente a 1,91\%, a mesma equivalência obtida com a otimização do sistema sob condições reais.

\section{CONCLUSÕES}

Com a elaboração de 4 cenários, aplicando-se as reduções de $25 \%$ e $50 \%$ na vazão de jusante do reservatório, de $25 \%$ no consumo de água urbano e posteriormente, $25 \%$ no consumo de água apenas das cidades que compõem o Tramo Norte, respectivamente, observou-se que diante destes, o melhor comportamento do sistema ocorreu no Cenário 3, tendo em vista que este apresentou um maior atendimento às demandas e um menor valor de redução para com as zonas urbanas, indicando uma redução de $79,29 \%$ para Caruaru. Para as áreas rurais, o resultado obtido em todos os cenários se manteve igual, apresentando déficit nas cidades do Tramo Sul. Os cenários elaborados não mostraram resultados positivos diante do cenário inicial. Sob condições reais, as soluções obtidas se mantiveram deficitárias quando comparadas com a otimização prioritária do sistema.

Portanto, mesmo o Cenário 3 sendo a situação que apresenta maior número de demandas atendidas, a melhor alternativa de funcionamento para o sistema, é a do cenário inicial da pesquisa, sem a aplicação de reduções nos valores de vazão e consumo, quando o sistema distribui uma vazão de 160 L/s, e é capaz de atender $100 \%$ do consumo total de água de 9 municípios, e ao consumo unicamente urbano de 6, apenas com uma redução de $98,09 \%$ para Caruaru. Sob estas condições, o SAABJ é otimizado, com um funcionamento mais eficaz do que o atual e com riscos menores de colapso, pois os valores que serão distribuídos, serão coerentes com a disponibilidade e capacidade do reservatório, porém é de grande importância destacar, que essa disponibilidade está prioritariamente relacionada aos índices pluviométricos da região.

Deve-se ressaltar que as variáveis de vazão consideradas para a realização da otimização foram teóricas, não foram com uma base de dados de medida e quantificação em loco, o que influencia nos resultados, principalmente em relação a zona rural, a área mais afetada. Dessa forma, pode-se indicar como solução eficaz para as áreas rurais das cidades do Tramo Sul, a implantação de um SAA integrado ao de Jucazinho, além de uma solução mediadora como a utilização de poços de extração de água. Como proposta para trabalhos futuros, uma pesquisa com maior preocupação referente as vazões, melhor detalhamento da rede do SAA e consideração do valor da vazão regularizada do rio Capibaribe, para a realização da otimização e obtenção de valores de maior precisão.

\section{REFERÊNCIAS}

ANDRADE SOBRINHO, R.; BORJA, P. C.. Gestão das perdas de água e energia em sistema de abastecimento de água da Embasa: um estudo dos fatores intervenientes na RMS. Eng. Sanit. Ambient., v.21, n.4, p.783-795, 2016.

APAC. Agência Pernambucana de Águas e Climas. Bacias Hidrográficas: Bacia do Capibaribe. Recife: APAC, 2018.

BRAZÃO, A. J. C.; SILVA, R. D. R.. Cenário do reuso de água no Nordeste brasileiro: estudos de casos e desafios. Natal: 2016.

COMPESA. Companhia Pernambucana de Saneamento. Barragem de Jucazinho. Recife: COMPESA, 2018.

DIEESE. Departamento Intersindical de Estatística e Estudos socioeconômicos. Visão geral dos serviços de água e esgotamento sanitário no Brasil: Estudos e pesquisas $n^{\circ} 82$. 
Recife: DIEESE, 2016

DUARTE, B. E. S.. Os sistemas de abastecimento d'água da grande João Pessoa e a espacialização das áreas abastecidas. Monografia (Graduação em Engenharia Civil) Universidade Federal da Paraíba, João Pessoa, 2016.

GOMES, H. P.. Sistemas de Saneamento: Eficiência energética. João Pessoa: UFPB, 2010.

IBGE. Instituto Brasileiro de Geografia e Estatística. Sinopse do Censo demográfico 2010 Pernambuco. Rio de Janeiro: IBGE, 2018.

MANSILHA, M. B.; FARRET, F. A.; KULLMANN, D. H.. Programação linear: método de otimização simplex e software otimiza. Revista Espacios, v.38, n.60, 2017.

MATTIUZI, C. D. P.. Gestão integrada dos recursos hídricos: alocação otimizada com uso conjunto de água superficial e subterrânea para redução da escassez hídrica na Bacia Do Rio Santa Maria/RS. Dissertação (Mestrado em Recursos Hídricos e Saneamento) - Universidade Federal do Rio Grande do Sul, Porto Alegre, 2018.

PEDROSA, H. T. S.. Otimização em estação elevatória de sistemas de abastecimento de água buscando a eficiência energética. Dissertação (Mestrado em Engenharia Civil) Universidade Federal de Pernambuco, Recife, 2015.

PEREIRA, L. S.. Abordagem multicritério para estabelecimento de prioridades gerenciais/comerciais para manutenção em sistemas de abastecimento com captação de água subterrânea. Dissertação (Mestrado em Engenharia de Produção) - Universidade Federal de Pernambuco, Recife, 2017.

PINTO, H. S.. A crise hídrica brasileira no contexto do plano para a segurança alimentar, nutrição e erradicação da fome 2025 da CELAC. Brasília, 2015.

SILVA, B. O.; FERREIRA, J. G.; SANTOS, R. T. L.. Dimensões da Governança da Água no Nordeste Brasileiro. Porto Alegre, 2017.

SILVA, D. C.; CANDIDO, G.; BARACUHY, J.; CHAVES, H.; CURI, W.. Gestão de recursos hídricos no Brasil e interdisciplinaridade: Uma reflexão em torno de apontamentos contemporâneos desta relação. Revista Espacios, v.38, n.1, 2017.

SILVA, S. F.; GENZ, F.; SILVA, N. M. D.; KIPERSTOK, A.. Avaliação da ocorrência de secas na Bahia utilizando o Índice de Precipitação Padronizada (SPI). Bahia Anál. Dados, Salvador, v.23, n.2, p.461-473, 2013.

SOUZA, L. M.. Gestão de Recursos Hídricos: Metodologias de Participação Social. Belo Horizonte, 2013.

SNIS. Sistema Nacional de Informações sobre Saneamento. Série Histórica: Água e esgoto. Brasília: SNIS 2018.

TCU. Tribunal de Contas da União. Relatório de auditoria: Obras paralisadas da Barragem de Jucazinho e das Barragens de Serrinha e Adutora do Oeste em Pernambuco. Brasília: TCU, 2000.

A CBPC - Companhia Brasileira de Produção Científica (CNPJ: 11.221.422/0001-03) detém os direitos materiais desta publicação. Os direitos referem-se à publicação do trabalho em qualquer parte do mundo, incluindo os direitos às renovações, expansões e disseminações da contribuição, bem como outros direitos subsidiários. Todos os trabalhos publicados eletronicamente poderão posteriormente ser publicados em coletâneas impressas sob coordenação da Sustenere Publishing, da Companhia Brasileira de Produção Científica e seus parceiros autorizados. Os (as) autores (as) preservam os direitos autorais, mas não têm permissão para a publicação da contribuição em outro meio, impresso ou digital, em português ou em tradução. 